\title{
Understanding priming of color-singleton search: Roles of attention at encoding and "retrieval"
}

\author{
BRIAN A. GOOLSBY and SATORU SUZUKI \\ Northwestern University, Evanston, Illinois
}

\begin{abstract}
We investigated how the performance of a color-singleton search (the search for a single odd-colored item among homogeneously colored distractors) left a persistent memory trace (lasting up to six intervening trials or $\sim 17 \mathrm{sec}$ ) that facilitated a subsequent color-singleton search (when the same targetdistractor color combination was repeated). Specifically, we investigated the roles of attention in the encoding and "retrieval" stages of this priming effect by intermixing trials in which the target location was precued by an onset cue. We found that the encoding of both target and distractor colors was automatic in that whether or not observers had to use color in locating the target in the preceding trial did not substantially affect priming. However, priming required that the color-singleton item be attended in the preceding trial. Once a color singleton display was encoded, our results indicated that priming facilitated the direction of attention to the color-singleton target on a subsequent trial. In short, when a color-singleton item happened to be a critical item to be attended in one situation, another color-singleton item defined by the same color combination tended to attract attention in subsequent encounters.
\end{abstract}

Color priming refers to the phenomenon in which the perceptual processing of a particular color facilitates subsequent processing of the same color. For example, a simple task of color classification (e.g., red and green in one category and blue and yellow in another category) is speeded when the same color is repeated in successive trials. Color priming has been dissociated from the effects of response priming (responses being speeded following the same response) and long-term response bias ${ }^{1}$ (e.g., Di Pace, Marangolo, \& Pizzamiglio, 1997; Simon, 1988).

Though these basic demonstrations of color priming are important, it is also interesting to examine color priming effects in a more realistic context. In particular, color is often useful for finding familiar objects. For example, a red apple can be easily spotted against green leaves of a tree on the basis of its red color. Color is a powerful feature for finding objects because it is often strongly associated with object identity (e.g., yellow banana) and is relatively unaffected by changes in illumination caused by shadows and shading. Not surprisingly, research has shown that the visual system has a mechanism that allows people to find a single odd-colored item (color sin-

This work was supported by a National Science Foundation Grant SBR-9817643 given to S.S. We thank Jeremy Wolfe, Jan Theeuwes, and an anonymous reviewer for constructive and helpful comments, and Doug Medin and Sania Hamilton for reading earlier versions of the manuscript. We are indebted to Marcia Grabowecky for helping to make the manuscript substantially shorter and clearer. A part of this work was presented at the 40th annual meeting of the Psychonomic Society (November, 1999). Correspondence concerning this article should be addressed to S. Suzuki, Northwestern University, Department of Psychology, 2029 Sheridan Rd., Evanston, IL 60208 (e-mail: satoru @ northwestern.edu). gleton) rapidly without having to attend to individualitems. For example, an odd-colored item can be spotted among homogeneously colored distractor items rapidly regardless of how many distractor items surround the oddcolored item (e.g., Bravo \& Nakayama, 1992; Treisman $\&$ Gelade, 1980). Furthermore, an irrelevant odd-colored item can automatically attract attention when observers search for a salient singleton target defined by another feature such as shape (e.g., looking for a circle among diamonds; Theeuwes, 1991, 1992; see Egeth \& Yantis, 1997, for a review). Given that color is important in the daily task of looking for objects and that the visual system can perform color-singleton searches rapidly, it is interesting to study color priming effects in the context of colorbased visual search.

Indeed, Maljkovic and Nakayama $(1994,2000)$ have recently demonstrated that detection of a color singleton is facilitated when a color singleton defined by the same color contrast was detected in the previous search trial relative to when the opposite color singleton was detected. For example, having searched for a red target among green distractors makes a subsequent search faster when a red target is again searched among green distractors compared with when a green target is searched among red distractors. This color-combination-specific priming of color-singleton detection cannot be attributed to a response bias or to a strengthening of a particular stimulusresponse ( $\mathrm{S}-\mathrm{R}$ ) connection because the observers' task was to make a shape discrimination about the target rather than to report the color of the target. Maljkovic and Nakayama (2000) suggested that the memory underlying this priming effect is "implicit" because their observers were unable to explicitly recall the color combination reliably 
beyond the immediately preceding trial, whereas the color priming effect from a single trial persisted through four to eight ${ }^{2}$ intervening trials in which the two color combinations occurred with equal probability.

In the present study, we investigated the processes underlying the encoding and "retrieval" aspects of this priming effect in some detail. To provide a conceptual framework, we speculated that, in performing the color-singleton search task, the following perceptual/cognitive processes were involved: (1) detecting colored items, (2) using color to locate and direct attention to the singleton item, (3) focusing attention on the color-singleton item, (4) identifying the shape of the color-singleton item, and (5) responding by pressing the appropriate computer key. To understand the encoding aspect of the priming effect, we attempted to determine which of these processes might be critical for laying a memory trace that facilitated a subsequent color-singleton search when the same colors were repeated. To understand the "retrieval" aspect (or the mechanism of facilitation) of the priming effect, we attempted to determine which of these processes might be facilitated by the priming effect.

Maljkovic and Nakayama (1994) found that although repetitions of the same color singleton produced robust priming effects, repetitions of the same shape (or response) did not. They reasoned that the priming facilitated the process of detecting the color-singleton target, but it did not facilitate the process of identifying the target shape or making the response. Furthermore, by referring to the phenomenon as priming of the attention-focusing feature ("priming of pop-out"), they implied that attending to color in finding the target (i.e., using color as the attentionfocusing feature) was critical in laying the appropriate memory trace for producing the priming effect. However, since their observers always had to use color in finding the target, this hypothesis was never tested against an alternative possibility that a color singleton might be encoded automatically, regardless of whether or not observers attended to color in finding the target. Previous studies on visual priming, though not for color priming or for visual search, have suggested that visual priming effects can be relatively unaffected by attention manipulations on the prime trial (e.g., Driver \& Tipper, 1989; Fox, 1994, 1995; see the General Discussion section for details). Nevertheless, since the process of directing attention to the target among distractors is a critical component in this search paradigm (requiring high-acuity shape discrimination on the target), attending to colors in finding the target in the prime trial may be critical for facilitating a subsequent color-singleton search.

We thus began by evaluating how priming might be affected when observers' attention was manipulated during a color-singleton search trial. We manipulated attention by interspersing Maljkovic and Nakayama's (1994) original trials with trials in which an onset cue preceded the stimulus array. The cue indicated the location of the upcoming target with $100 \%$ validity. Such an onset cue has been shown to automatically draw observers' attention to the cued location within 100 to $200 \mathrm{msec}$ (e.g., Cheal
\& Lyon, 1989; Eriksen \& Collins, 1969; Eriksen \& Rohrbaugh, 1970; Mackeben \& Nakayama, 1993; Nakayama \& Mackeben, 1989; Suzuki \& Cavanagh, 1997). Thus, assuming that our onset cue effectively attracts attention to the target (obviating the process of attending to color in finding the target), we considered the following outcomes.

If the priming of color-singleton search requires that color be attended in finding the target in a preceding trial, prior cued trials should produce substantially reduced (or no) priming on subsequent color-singleton search trials. Alternatively, if the priming occurs automatically irrespective of whether or not observers perform a colorbased search in a preceding trial, prior cued trials and prior search trials should produce a comparable degree of priming on subsequent color-singleton search trials.

We further evaluated the possibility that eliminating the distractors (Experiment 2) might be equivalent to precuing the target location (Experiment 1 ). If a location cue reduces or eliminates encoding of the distractors, prior target-alone (no distractors) trials should produce as much (or as little) priming as prior cued trials on subsequent search trials. However, if encoding of the distractors is necessary for priming, prior target-alone trials should not produce priming.

In Experiment 3, we evaluated the possibility that the priming of color-singleton search might be a passive repetition priming effect-a consequence of perceiving a particular color singleton. We reasoned that, if the priming effect was fully automatic, detection of, for example, a green singleton among red distractors should be facilitated when it is preceded by a display containing a green singleton among red distractors, whether or not observers attend to the green singleton in the preceding trial.

Across these experiments, our first goal was consideration of the encoding aspect of the priming effect by manipulating prior trials and examining how their priming of subsequent trials was affected. Our second goal was to understand how priming facilitates color-singleton search by examining the effects of manipulating current trials. If color-singleton priming facilitates search, priming should be substantially reduced on current cued trials. This is because priming should be preempted by the onset cue, which should focus observers' attention to the target location. Alternatively, the mechanism of the priming effect might be unrelated to directing attention to the target. For example, shape discrimination might be facilitated when it is done repeatedly in the same color context. If priming facilitates this and other processes unrelated to focusing attention to the target, it should continue to be present on the current cued trials. Finally, even if the onset cue facilitates the focusing of attention to the repeated color singleton, if the onset cue and the priming focused attention through separate mechanisms, the priming effect could be additive with the effect of the onset cue.

\section{GENERAL METHOD}

\section{Observers}

All observers had normal or corrected-to-normal vision and were tested individually in a dimly lit room. The 2 authors and 13 paid 
observers (graduate and undergraduate students at Northwestern University, who were naive to the purpose of the experiments) participated in Experiments 1A, 1B, 2, and 3; the first author participated in all experiments except Experiment $1 \mathrm{C}$; the second author participated in Experiments 1A, 2, and 3; 1 of the naive observers participated in Experiments 1A and 2; another participated in Experiments $1 \mathrm{~A}$ and 3 ; each of the remaining 11 observers participated in only one of the four experiments. ${ }^{3}$ In addition, 28 undergraduate students from Northwestern University were recruited for Experiment $1 \mathrm{C}$; they participated for a course requirement.

\section{Apparatus}

Stimuli were displayed on a 17 -in. color monitor $(75 \mathrm{~Hz})$ and all experiments were controlled with a Macintosh PowerPC 8600 (300 MHz) computer using the Vision Shell software (micro ML, Inc., Quebec, Canada).

\section{Stimuli}

The standard display consisted of three diamond shapes arranged on an imaginary ellipse ${ }^{4}$ centered at the fixation point (Figure 1A). The horizontal axis of the ellipse subtended $10.1^{\circ}$ visual angle and the vertical axis subtended $8.2^{\circ}$. The diamond shapes could be presented at any of the 12 possible locations along the circumference of this imaginary ellipse (at $0^{\circ}, 30^{\circ}, 60^{\circ}, 90^{\circ}, 120^{\circ}, \ldots, 300^{\circ}$, and $330^{\circ}$ locations beginning at the top of the vertical meridian) with the constraint that the three diamonds were approximately equidistant from each other- that is, separated from each other by four location steps (or by $120^{\circ}$ rotation). Each diamond subtended $1.3^{\circ} \times$ $1.3^{\circ}$ visual angle and had the left or right side "chipped" with the depth of chip subtending $0.22^{\circ}$. The side of the chip for each diamond was randomly determined in each trial.

In each stimulus display, one of the three diamonds had a different color than the other two diamonds-a red diamond among two green diamonds or a green diamond among two red diamonds. The location and color of the odd-colored diamond were determined randomly in each trial. The red (CIE[.629, .346]) and the green (CIE[.299, .598]) used were set to be approximately equiluminant for each observer, as determined by flicker photometry at a frequency of $20 \mathrm{~Hz}$. The red had a fixed luminance of $5.0 \mathrm{~cd} / \mathrm{m}^{2}$, and the green had an adjusted luminance ranging from 4.7 to $6.6 \mathrm{~cd} / \mathrm{m}^{2}$ for individual observers. The stimuli were presented against a dark background (all color guns on the monitor turned off).

The fixation marker presented at the center of the screen was a small achromatic open circle $\left(39 \mathrm{~cd} / \mathrm{m}^{2}\right.$, CIE[.262, .282], diameter $=$ $.26^{\circ}$ ) drawn with a one-pixel-thick line (each pixel subtending $0.043^{\circ}$ ); the fixation marker remained on throughout each trial. In Experiments $1 \mathrm{~A}, 1 \mathrm{~B}, 1 \mathrm{C}$, and 3 , half of the trials were preceded by an onset cue. The onset cue was an open circle (same luminance and color as the fixation point) with diameter $1.94^{\circ}$ (about $50 \%$ larger than the diameter of the diamonds) drawn with a one-pixel-thick line (Figure 1B).

Observers were seated in a hard-backed chair at $50 \mathrm{~cm}$ from the screen. Although head position was not fixed with a chinrest, the observers measured and adjusted their viewing distance before the beginning of each block of 200 trials.

\section{Procedure}

Each trial began with a blank fixation screen presented for a variable interval of $2-2.5 \mathrm{sec}$, followed by a stimulus display. A standard color-singleton display consisted of the three diamond shapes, one of which was oddly colored; in most cases, the odd-colored item was the target item for shape discrimination (except for the cued trials in Experiment 3, in which a noncolor-singleton item was the target). In some cases (Experiment $1 \mathrm{C}$ and half of the trials in Experiment 2), the display consisted of only one diamond shape (the target item). The stimulus display remained on until the observer made a response. The observers reported the side of chip on the target item by making a keypress; they pressed the " $z$ " key (located on the lower left side of keyboard) with the left index finger to report a left-side chip and pressed the "/" key (located on the lower right side of keyboard) with the right index finger to report a right-side chip. Thus, the locations of the response keys were compatible with the sides of chip reported. The observers were instructed to respond as quickly as possible while preserving accuracy above $95 \%$. The computer automatically recorded the response time and response key for each trial. No feedback was given for incorrect responses.

The observers were instructed to maintain eye fixation on the fixation marker prior to the presentation of each stimulus array. As the shape discrimination required a high acuity judgment, eye movements to the target were allowed after the onset of the stimulus array (as was the case in Maljkovic \& Nakayama's, 1994, study).

In Experiments 1A, 1C, and 3, half of the trials were preceded by the circular onset cue flashed at the location of the target item; the circular cue was presented centrally in Experiment 1B so that it served only as a temporal cue. The onset cue had a duration of $27 \mathrm{msec}$ and preceded the stimulus array by a stimulus-onset asynchrony (SOA) of $148 \mathrm{msec}$. As discussed in the introduction, this particular SOA was chosen because previous studies using various measures (e.g., response time, vernier acuity, and spatial distortion effects) have demonstrated that an onset cue attracts attention to the cued location maximally when cue-to-stimulus SOA is 100 $250 \mathrm{msec}$. Cued trials were randomly intermixed with noncued trials. The observers were told that the position cue, whenever present, always indicated the location of the target item (100\% valid).

All observers except for the authors were given one block of practice trials during the initial orientation session-200 trials for Experiments 1A, 1B, 2, and 3, and 10 trials for Experiment 1C. In Experiments $1 \mathrm{~A}, 1 \mathrm{~B}, 2$, and 3, each observer was tested extensively, 2,000-4,800 trials per experiment. The trials were given in several experimental sessions; a typical session (lasting about $1 \mathrm{~h}$ ) consisted of four blocks of 200 trials; short breaks were allowed between blocks. The exact number of trials depended on the number of experimental conditions and the random variability in the data, which depended on both the nature of the task and individual differences. Additional trials were run whenever necessary so that the baseline priming effect (replication of Maljkovic \& Nakayama, 1994), against which the experimental effects were evaluated, was clearly seen above the random (unexplained) variability for each observer.

In Experiment $1 \mathrm{C}$, in which a large number of observers participated, each observer was tested in 200 trials.

\section{Data Analysis}

Only RTs for trials on which correct responses were made were used in the analyses. The average error rate across all experiments was low $(<3 \%)$. For those experiments in which each observer was tested extensively (Experiments 1A, 1B, 2, and 3), trimmed mean RTs (using three standard deviations as the cutoff) were computed for the smallest experimental cells for each observer; the percentage of trials trimmed was less than $2 \%$ across all observers for each of the four experiments. For Experiment 1C, in which each observer contributed many fewer trials, median ${ }^{5}$ RTs were obtained for the smallest experimental cells for each observer. All statistical analyses were conducted using observer as the random variable.

\section{EXPERIMENT 1A Effects of Precuing the Target Location on the Priming of Color-Singleton Search}

The aim of this experiment was twofold. First, we examined how precuing the target location affected the encoding of colors underlying the priming effect. Specifically, we examined whether the precuing of the target location in a prior trial reduced (or eliminated) priming of subsequent color-singleton search trials (relative to prim- 
A
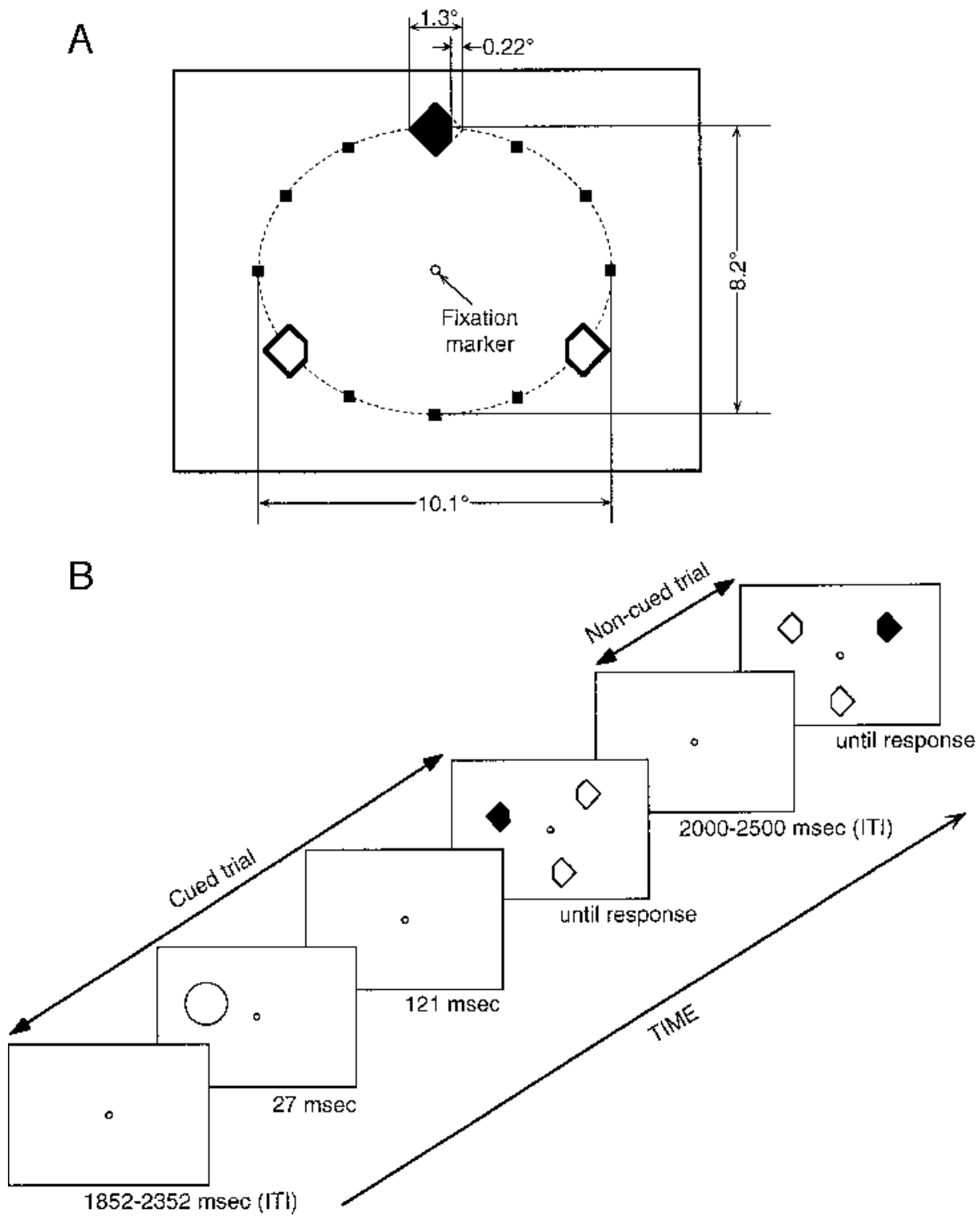

Figure 1. (A) The 12 possible target locations (small squares) are shown with an example of a target (the filled figure) and two distractors (open figures). To keep the visual acuity approximately equal for each stimulus location, the stimuli were placed on an imaginary ellipse. The target could appear at any of the 12 locations; the two distractors were always separated by four location steps (or $120^{\circ}$ rotation) from the target and from each other. (B) A schematic trial sequence of a cued trial (a color-singleton trial preceded by a position cue) followed by a search trial (a color-singleton trial without the position cue). The interval between successive stimulus displays were randomly varied between 2 and 2.5 sec to prevent observers from anticipating the onset of the stimulus display. On cued trials, this blank intertrial interval was shortened by the length of the cue-to-stimulus stimulus onset asynchrony $(148 \mathrm{msec})$ so that the time interval between the successive stimulus displays was unaffected by the cues.

ing produced by prior search trials). Second, we examined how precuing within the current trial affected its priming from prior trials.

\section{Method}

Observers. Seven observers (the 2 authors and 5 naive observers) participated.
Stimuli and Procedure. The observers were instructed to find the odd-colored item (target) and to report which side of it was chipped. In half of the trials, the target location was precued (cued trials), and, in the rest of the trials, the target location was not precued (search trials). The cued trials and search trials were randomly intermixed. The observers were told that, when present, the cue would always identify the location of the upcoming color-singleton target (cue validity $=100 \%$ ). Observers completed 2,000-4,800 trials. 


\section{Results and Discussion}

Method of data analysis. We examined the priming effect of a single trial on subsequent trials across 0-14 intervening trials; this gave us a sufficient range to be able to determine the duration of the priming effect based on Maljkovic and Nakayama's (1994) finding that the priming effect persisted up to eight trials.

We examined the effects of repeating the same target and distractor colors - color priming and the effects of repeating the same target chip (and the same response) chip/response priming. For color priming, the priming effect produced by the $k$ th trial back was measured by subtracting the mean RT of all trials for which the color combination (red target among green distractors or green target among red distractors) was different the $k$ th trial back from the mean RT of all trials for which the color combination was the same the $k$ th trial back.

Priming effect (produced by the $k$ th trial back) $=$ [mean RT of all trials for which the $k$ th trial back had the same color combination] - [mean RT of all trials for which the $k$ th trial back had a different color combination].

Thus, a negative value indicates that the preserved color combination across $k-1$ intervening trials (in which the color combination changed randomly) reduced RT relative to when the color combination was switched.

Since each trial could be a search trial or a cued trial, there were four combinations of priming: (1) priming from a prior search trial on a subsequent search trial, (2) priming from a prior cued trial on a subsequent search trial, (3) priming from a prior search trial on a subsequent cued trial, and (4) priming from a prior cued trial on a subsequent cued trial. These four types of priming were examined separately. The color priming effect of a prior search trial (or a prior cued trial) on a subsequent search trial was assessed by averaging the RTs of search trials for which the $k$ th trial back was a search trial (or a cued trial). Similarly, the priming effect of a prior search trial (or a prior cued trial) on a subsequent cued trial was assessed by averaging the RTs of cued trials for which the $k$ th trial back was a search trial (or a cued trial).

The chip/response priming (the effect of whether the side of chip was the same or different $k$ trials back) was evaluated similarly. However, since the chip/response priming was not the focus of this article, we do not present its analysis in detail or for every experiment. The chip/ response priming data will be presented only in cases where it is relevant to the understanding of the priming of color-singleton search.

Previous results (Maljkovic \& Nakayama, 1994) indicated that the color-singleton priming effect examined here was greatest for immediate repetitions of a samecolored trial and monotonically decayed as more intervening trials were inserted. We therefore adopted a method of sequential $t$ tests (2-tailed) for assessing the duration of the priming effect (across the number of trials it persisted). If the priming effect produced by the 1 st trial back was not significant (not significantly different from zero), we concluded that there was no significant prim- ing effect. If it was significant, we then performed a $t$ test on the priming effect produced by the 2 nd trial back. If it was not significant, we concluded that the priming effect lasted one trial only, whereas, if it was significant, we performed a $t$ test on the priming effect produced by the 3rd trial back, and so on. In general, if the priming effect was significant for the 1 st trial back through the $k$ th trial back, we concluded that the priming lasted $k$ trials.

Color priming from prior search trials and prior cued trials on subsequent search trials. In Figure $2 \mathrm{~A}$, the priming effects (color-same RT minus color-different RT) produced by the 1 st trial back through the 15 th trial back when the current trial was a search trial are plotted. As can be seen in the figure, substantial color priming was obtained on a search trial, regardless of whether it was preceded by a search trial (squares) or a cued trial (circles). The data have been averaged across the 7 observers, but all observers showed the same pattern of results.

The priming effect produced by a preceding search trial lasted over five trials (filled squares in Figure 2A) [1 st trial back, $t(6)=5.466, p<.005 ; 2$ nd trial back, $t(6)=4.638, p<.005 ; 3$ rd trial back, $t(6)=4.404, p<$ .005 ; 4 th trial back, $t(6)=3.709, p<.01$; and 5 th trial back, $t(6)=3.313, p<.05]$. The priming effect produced by a preceding cued trial lasted three to five trials (filled circles in Figure 2A) [1 st trial back, $t(6)=4.869$, $p<.005 ; 2$ nd trial back, $t(6)=3.447, p<.05$; 3rd trial back, $t(6)=2.745, p<.05$; marginally significant at the 4th trial back, $t(6)=2.307, p<.07$; and 5 th trial back, $t(6)=3.197, p<.05]$.

The strong color priming effect produced by a prior search trial on a subsequent search trial replicated the basic result of Maljkovic and Nakayama (1994). The almost equally strong priming effect produced by a prior cued trial on a subsequent search trial suggests that the encoding of colors underlying the priming effect occurred even when the target location was precued-that is, even when observers did not need to use color to find the target.

Note, however, that the priming effect produced by a prior cued trial was slightly less than that produced by a prior search trial (Figure 2A). When the priming data were pooled across the significant range (the 1 st through the 5 th trial back), the difference in priming was significant [mean difference $=9 \mathrm{msec}, S E=2 \mathrm{msec}, t(6)=5.226$, $p<.005]$. Thus, although a prior cued trial produced a reliable priming effect, its magnitude was slightly less than that produced by a prior search trial.

We further examined whether the reduction in priming was due to a reduced benefit of color repetition or to a reduced cost of color switching. This was done by comparing the mean RTs when search trials were: (1) preceded by color-different search trials, (2) preceded by color-same search trials, (3) preceded by color-different cued trials, and (4) preceded by color-same cued trials. As above, the RT data were pooled across the significant range of the priming effect (the 1 st through the 5 th trial back). As can be seen in Figure 3, the mean RTs for the color-different condition were identical when the preceding trial was a search trial $(612 \mathrm{msec})$ and when it was a cued trial 

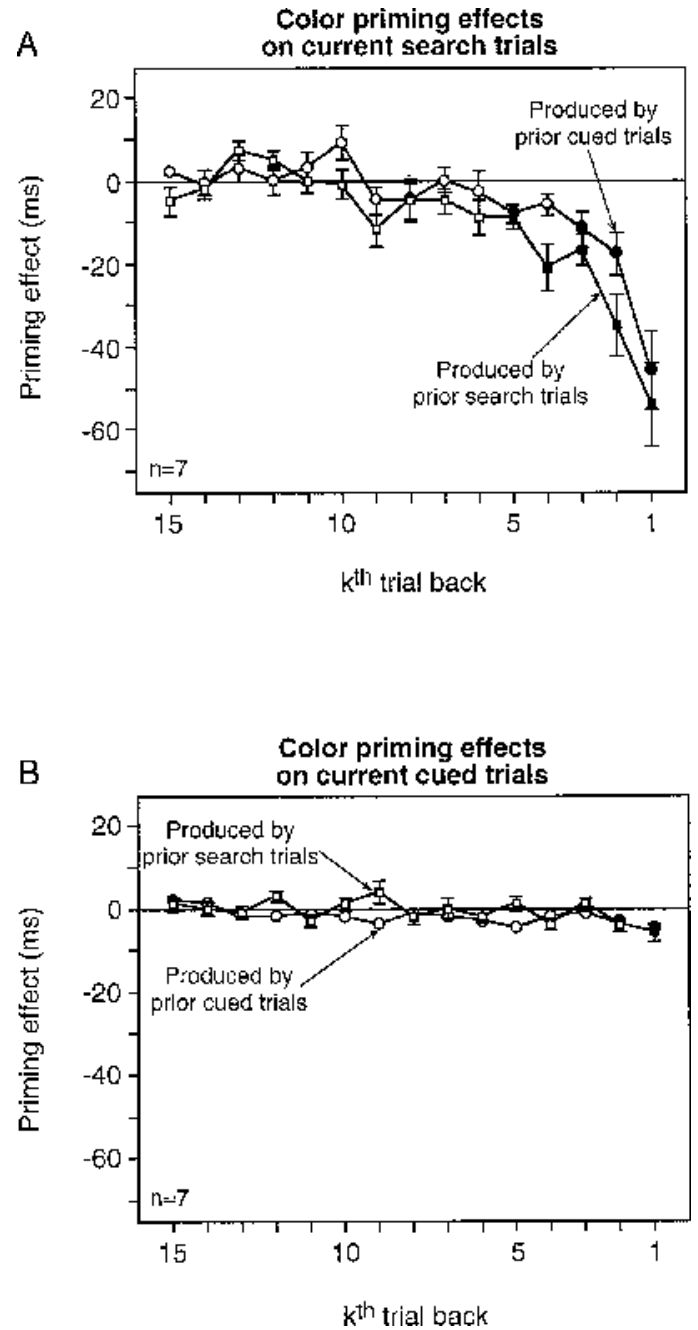

Figure 2. Color priming effects (mean RT of trials for which color combination was the same the $k$ th trial back minus mean RT of trials for which color combination was different the $k$ th trial back) obtained in Experiment 1A. (A) Priming effects on current search trials produced by prior search trials (squares) and prior cued trials (circles). (B) Priming effects on current cued trials produced by prior search trials (squares) and prior cued trials (circles). Filled symbols indicate that the priming effect was significantly different from zero ( $p<.05$, two-tailed). Error bars represent $\pm 1 S E$ (observers as the random effect).

(612 msec). In contrast, the mean RTs for the color-same condition were slower when preceded by a cued trial $(594 \mathrm{msec})$ than when preceded by a search trial $[585 \mathrm{msec}$, $t(6)=5.375, p<.005]$. The data thus suggest that the slightly reduced priming effect produced by a prior cued trial was due to reduced benefit of color repetition.

The main finding so far is that prior precued trials produced persistent priming effects on subsequent search trials, suggesting that using color in finding the target was not required for the color encoding that underlies the priming effect. The magnitude of the priming produced by the prior precued trials, however, was modestly but significantly less than that produced by the prior search trials. One interpretation would be that precuing slightly weakened the encoding of relevant colors because the observers did not need to attend to color in finding the target. Alternatively, any flashed circle preceding the colorsingleton display might interfere with encoding of the relevant colors and thereby reduce priming on subsequent trials. The latter possibility was tested in Experiment 1B by presenting the cue circle at the center of the display. If precuing the target location reduced encoding of the relevant colors because the observers did not need to attend to color in finding the target, then a centrally presented circle should not reduce subsequent priming. Alternatively, if the cue interfered with color encoding simply by virtue of flashing, the presentation of a centrally flashed circle should also weaken subsequent priming.

Color priming from prior search trials and prior cued trials on subsequent cued trials. As is shown in Figure 2B, little priming was obtained on a current cued trial, whether it was preceded by a search trial or by a cued trial. Though small in magnitude, the priming effect produced by a prior search trial (filled squares in Figure $2 \mathrm{~B}$ ) was significant at the 1 st trial back $[t(6)=2.635$, $p<.05]$ and the priming effect produced by a prior cued trial (filled circles in Figure 2B) was significant at the 1 st and 2 nd trial back [ 1 st, $t(6)=6.562, p<.001 ; 2 \mathrm{nd}$, $t(6)=3.422, p<.05]$. The priming effects on the current cued trials ( $6 \mathrm{msec}$ on average at the 1 st trial back) were rather small, relative to the priming effects on the current search trials (50 msec on average at the 1 st trial back).

This dramatic reduction of priming on the currently cued trials is consistent with an interpretation that the

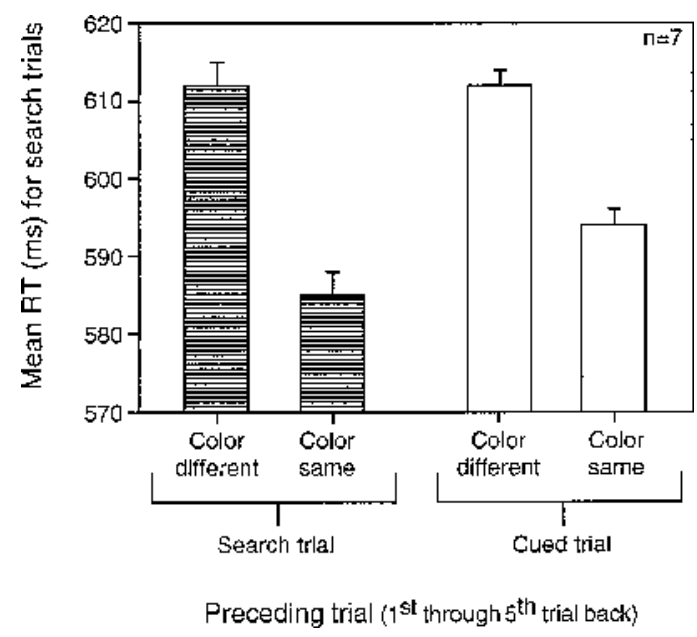

Figure 3. An analysis of mean RTs for Experiment 1A. Mean RTs for current search trials are plotted under four conditions: (1) preceded by a color-different search trial, (2) preceded by a color-same search trial, (3) preceded by a color-different cued trial, and (4) preceded by a color-same cued trial (from left to right; the effects from the 1 st trial back through the 5 th trial back have been pooled; see text for details). Error bars represent $1 S E$ (The variance due to differences in baseline $R T$ among the observers has been removed). 
onset cue preempted priming. This in turn implies that the effect of priming was to facilitate the process of drawing attention to the target. However, alternative possibilities need to be evaluated. For example, analogous to our argument for a possible effect of any flashed circle on color encoding, any flashed circle immediately preceding a search display might also disrupt priming from prior trials. Experiment $1 \mathrm{~B}$, in which the cue circle was presented at the center of the display, allowed us to test both of these possibilities.

In addition, a floor effect might also have contributed to the much reduced priming effects on the current cued trials. RTs for the cued trials were substantially faster than those for the search trials. Therefore, the priming effects on cued trials might not have been measurable as reduced RTs, because the $100 \%$ valid position cue maximally facilitated performance on those trials. Evidence against this possibility was provided by the chip/response priming effect.

To examine the overall chip/response priming effects on current search trials and current cued trials, we averaged priming across the prior search trials and the prior cued trials. Since the chip/response priming effects were small, ${ }^{6}$ averaging allowed us to compare between priming on current cued trials and current search trials with greater statistical power. As can be seen in Figure 4, the chip/response priming effects were nearly identical on the current search trials and the current cued trials (if anything, longer lasting on the current cued trials). The chip/response priming effects on current search trials were not significant at the 1 st trial back $[t(6)=0.681$, n.s. $]$, but were significant at the 2 nd trial back $[t(6)=3.068, p<$ $.05]$, and marginally significant at the $3 \mathrm{rd}$ trial back $[t(6)=2.314, p<.06]$. The chip/response priming effects on current cued trials were marginally significant at the 1 st trial back $[t(6)=2.191, p<.08]$ and significant at the 2 nd through the 7 th trial back [2nd, $t(6)=3.448$, $p<.05 ; 3 \mathrm{rd}, t(6)=5.901, p<.005 ; 4$ th $, t(6)=6.070, p<$ $.001 ; 5$ th $, t(6)=5.453, p<.005 ; 6$ th $, t(6)=4.131, p<.01$; and 7 th, $t(6)=3.179, p<.05]$.

The fact that the chip/response priming effects did not diminish on the current cued trials ${ }^{7}$ suggests that the RTs on the cued trials were not at the floor. The evidence against the floor effect hypothesis was replicated and extended in Experiment 1C.

The effect of position cuing on RTs. All of the arguments above depend on the assumption that the onset cue effectively drew attention to the target. Indeed, the responses on the cued trials $(M=433 \mathrm{msec}, S E=25 \mathrm{msec})$ were substantially faster than those on the search trials ( $M=601 \mathrm{msec}, S E=15 \mathrm{msec}$ ), yielding a large cuing effect of $168 \mathrm{msec}[t(6)=10.516, p<.0001]$. However, it is possible that the large reduction in RT on the cued trials was mainly due to a temporal warning effect (e.g., Shulman, Remington, \& McLean, 1979; Ulrich \& Mattes, 1996). Since the intertrial interval (ITI) was varied between 2 and $2.5 \mathrm{sec}$, the cue could have allowed the observers to anticipate the onset of the stimulus display, and thus prepared them to make a response. This possibility was also tested in Experiment 1B using a centrally presented circle.

\section{EXPERIMENT 1B \\ A Control Experiment Using a Centrally Flashed Circle Instead of a Position Cue}

The aim of this experiment was threefold. First, if the large cuing effect obtained in Experiment $1 \mathrm{~A}$ was mainly due to a temporal warning effect, a centrally presented circle (which conveyed temporal information about stimulus onset but provided no information about target location) would be equally effective in facilitating RTs. Second, the prior cued trials in Experiment 1A produced relatively weaker priming effects (compared with the prior search trials) on subsequent search trials. If this was because flashed circles interfered with the process of encoding the relevant colors, prior trials with a centrally flashed circle should also produce weaker priming than should prior search trials. Third, if any flashed circle disrupts the current operation of priming, priming should be substantially reduced (or eliminated) on current trials with a centrally flashed circle, as well as on current positioncued trials (Experiment 1A).

\section{Method}

Observers. Five observers (the first author and 4 naive observers) participated.

Stimuli and Procedure. The stimuli and procedure were identical to those in Experiment 1A, except that the onset cue circle was centered at the fixation point rather than at the target location. Observers completed 2,000-2,400 trials.

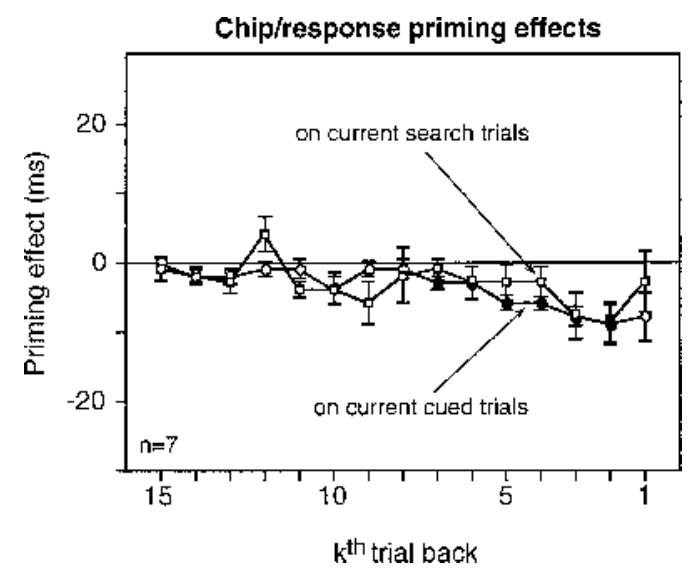

Figure 4. Chip/response priming effects (mean RT of trials for which chip side was the same the $k$ th trial back minus mean RT of trials for which chip side was different the $k$ th trial back) obtained in Experiment 1A. Priming effects on current search trials (squares) and current cued trials (circles) are shown; the priming effects have been collapsed over those produced by prior search trials and prior cued trials. Filled symbols indicate that the priming effect was significantly different from zero $(p<.05$, two-tailed $)$. Error bars represent $\pm 1 S E$ (observers as the random effect). 


\section{Results and Discussion}

The effect of centrally flashed circle on RTs. The centrally flashed circle provided no RT benefits $[M=$ $653 \mathrm{msec}, S E=48 \mathrm{msec}$ with the flashed circle vs. $M=$ $647 \mathrm{msec}, S E=45 \mathrm{msec}$ without the flashed circle; $t(4)=0.801$, n.s.]. It is thus likely that the onset cue used in Experiment 1A reduced RT by prefocusing observers' attention on the target rather than by a temporal warning effect. It is therefore reasonable to assume that the position cue used in Experiment 1A substantially reduced the need for attending to color in finding the target.

Color priming from prior search trials (with and without a centrally flashed circle) on subsequent search trials (without a centrally flashed circle). Prior search trials with or without a centrally flashed circle produced equivalent priming on subsequent search trials that were without a centrally flashed circle (Figure 5A). The priming effect produced by a prior search trial without the flashed circle lasted four trials (filled squares in Figure 5A) [1 st trial back, $t(4)=7.319, p<.005 ; 2$ nd trial back, $t(4)=3.827, p<.05 ; 3$ rd trial back, $t(4)=$ $3.608, p<.05$; and 4th trial back, $t(4)=17.273, p<$ $.0001]$. The priming effect produced by a prior search trial with the circle lasted six trials (filled circles in Figure $5 \mathrm{~A}$ ) $[1$ st trial back, $t(4)=7.235, p<.005 ; 2$ nd trial back, $t(4)=5.927, p<.005 ; 3$ rd trial back, $t(4)=$ $2.957, p<.05 ; 4$ th trial back, $t(4)=3.922, p<.05 ; 5$ th trial back, $t(4)=2.765, p<.05$; and 6th trial back, $t(4)=3.113, p<.05]$. These results are consistent with the idea that the position-cued trials in Experiment 1A produced slightly weaker priming effects on subsequent search trials, not because any flashed circle would interfere with encoding of the relevant colors, but because precuing the target location (thereby bypassing the process of attending to colors in finding the target) somewhat weakened color encoding. 8

Color priming from prior search trials (with and without a centrally flashed circle) on subsequent search trials (with the centrally flashed circle). The priming effects on the current search trials with the centrally flashed circle (Figure 5B) were robust and comparable to the priming effects on the current search trials without the centrally flashed circle (Figure 5A), whether the preceding trial was with or without the centrally flashed circle. When preceded by a search trial without the circle, the priming effect on the current search trials with the flashed circle lasted five trials (filled squares in Figure 5B) [1 st trial back, $t(4)=7.887, p<.005 ; 2$ nd trial back, $t(4)=4.644, p<.01$; 3rd trial back, $t(4)=$ $5.792, p<.005 ;$ th trial back, $t(4)=3.598, p<.05$; and 5 th trial back, $t(4)=4.219, p<.05]$. When preceded by a search trial with the circle, the priming effect lasted six trials (filled circles in Figure 5B) [1 st trial back, $t(4)=$ $6.100, p<.005 ; 2$ nd trial back, $t(4)=8.203, p<.005$; 3rd trial back, $t(4)=8.081, p<.005 ; 4$ th trial back, $t(4)=6.859, p<.005 ; 5$ th trial back, $t(4)=3.491, p<$ .05 ; and 6th trial back, $t(4)=8.557, p<.001]$. Thus, the mere presence of a flashed circle immediately preceding
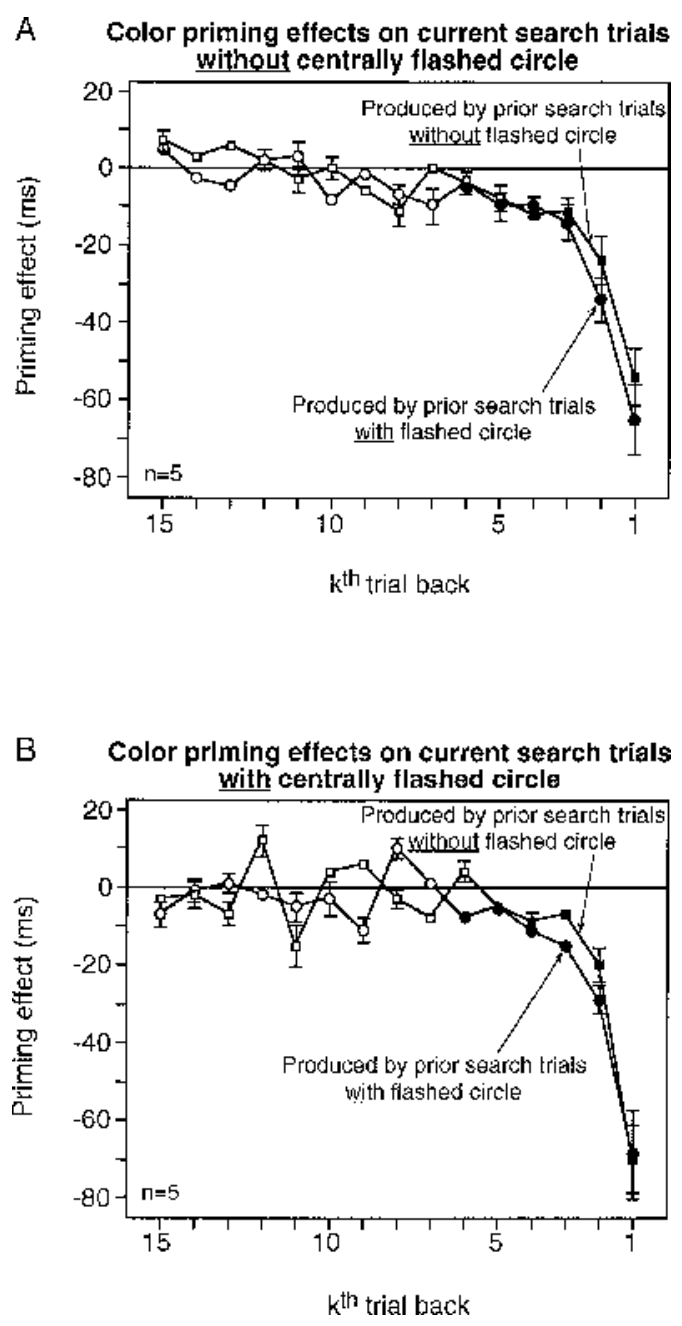

Figure 5. Color priming effects obtained in Experiment $1 \mathrm{~B}$. (A) Priming effects on current search trials without the centrally flashed circle produced by prior search trials without (squares) and with (circles) the centrally flashed circle. (B) Priming effects on current search trials with the centrally flashed circle produced by prior search trials without (squares) and with (circles) the centrally flashed circle. Filled symbols indicate that the priming effect was significantly different from zero $(p<.05$, two-tailed). Error bars represent $\pm 1 S E$ (observers as the random effect).

the stimulus array cannot explain why the priming effects were nearly eliminated on the current cued trials in Experiment $1 \mathrm{~A}$. The next experiment evaluated the RT floor effect hypothesis discussed above.

\section{EXPERIMENT 1C An Evaluation of a Possible RT Floor Effect on Cued Trials}

The goal of this experiment was to show that the position cuing used in Experiment $1 \mathrm{~A}$ specifically preempted the color-singleton priming effect instead of reducing the general RT to the floor. We found in Experiment $1 \mathrm{~A}$ that 
the chip/response priming effects were undiminished on the current cued trials. However, this finding needed to be replicated partly because the chip/response priming effects were generally weak and partly because we were trying to accrue evidence in support of a null hypothesis. To test the floor effect hypothesis rigorously, we made the task easier by presenting the target item alone without distractors-target-alone trials. So long as validly precuing the target location did not produce a floor effect, the chip/response priming should remain equally strong on the current cued trials and on the current noncued trials.

Furthermore, if we were correct in hypothesizing that color-singleton priming facilitated the attraction of attention to the target, little priming would be expected on either current cued or current noncued trials in the absence of distractors. This is because in the target-alone trials, the target would strongly capture attention by virtue of being the only onset item in the display.

\section{Method}

Observers. Twenty-eight observers ${ }^{9}$ participated. One observer was removed from the analysis due to inconsistent use of the response keys.

Stimuli and Procedure. The stimuli and procedure were identical to those used in Experiment 1A, except that the target was presented alone without distractors. As in Experiment 1A, the target location was precued in half of the trials. Each observer was tested in 200 trials. $^{10}$

\section{Results and Discussion}

The mean RT was significantly faster on the cued trials $(M=508 \mathrm{msec}, S E=10 \mathrm{msec})$ than on the noncued trials $(M=574 \mathrm{msec}, S E=10 \mathrm{msec})[t(26)=18.589$, $p<.0001]$.

Since the purpose of this experiment was to evaluate a potential floor effect on the cued trials, we collapsed over the priming produced by the prior cued and prior noncued trials and compared priming on subsequent cued trials with that on subsequent noncued trials.

As expected, little color priming was obtained on either current cued or current noncued trials (Figure 6A). Nevertheless, a small color priming effect on the current noncued trials appeared at the 1 st trial back $[t(26)=$ $2.082, p<.05]$. In contrast, chip/response priming effects were obtained on both the current cued and noncued trials (Figure 6B). The priming effect on the current noncued trials lasted three trials [ 1 st trial back, $t(26)=2.456$, $p<.05 ; 2$ nd trial back, $t(26)=7.366, p<.0001$; and 3rd trial back, $t(26)=2.750, p<.05]$. The priming effect on the current cued trials lasted three to five trials [ $1 \mathrm{st}$ trial back, $t(26)=6.547, p<.0001 ; 2$ nd trial back, $t(26)=$ $4.576, p<.0005$; 3rd trial back, $t(26)=7.087, p<.0001$; marginally significant at the 4 th trial back, $t(26)=1.839$, $p<.08$; and 5th trial back, $t(26)=5.044, p<.0001]$.

Thus, position cuing did not diminish the chip/response priming even when the task was made easier by presenting the target without distractors. This supports the idea that the position cuing used in Experiment $1 \mathrm{~A}$ did not reduce RT to the floor.

\section{Conclusions from Experiments 1A, 1B, and 1C}

The result of Experiment 1B ruled out the possibility that the precue acted merely as a temporal warning. It is thus reasonable to assume that the onset cue used in Experiment 1 A facilitated RT by focusing observers' attention at the target location and thereby obviated the process of search by color. Experiment $1 \mathrm{~B}$ also showed that the presence of a flashed circle per se (without signaling the location of the target) interfered neither with the encoding of colors underlying the priming effect nor with the operation of the priming effect. Furthermore, Experiment $1 \mathrm{C}$ provided evidence against the possibility that precuing the target location caused a general RT floor effect. Taken together, the results of these control experiments suggested that the modulation of the priming effect produced by the precuing procedure used in Experiment $1 \mathrm{~A}$ was due to the fact that the cue prefocused observers' attention on the target location.

We were thus able to interpret the results of Experiment $1 \mathrm{~A}$ with respect to the encoding requirements and the mechanism of the color-singleton priming effect. First, the fact that the priming effects on current search trials were reliable, regardless of whether the target location was validly precued or not in the preceding trial, suggested that encoding of the relevant colors was strong whether observers attended to colors in finding the target or their attention was prefocused on the target by the precue. Therefore, the process of attending to color in finding the target was not required for encoding color. However, the fact that precued trials produced slightly (but significantly) weaker priming suggested that not having to attend to colors in finding the target weakened encoding of the relevant colors (but see note 8). Second, the fact that precuing the target location virtually eliminated priming on the current cued trials - that is, the fact that priming little affected RT when observers' attention was prefocused on the target-suggested that priming facilitated the process of directing attention to the color-singleton target.

In Experiments 2 and 3, we investigated the encoding process further. In Experiment 2, we evaluated the possibility that priming was driven primarily by encoding of the target color (not by encoding of the distractor color). If so, precuing the target location would leave unaffected the necessary color encoding underlying priming of singleton search (consistent with Experiment 1A).

\section{EXPERIMENT 2 \\ Is Encoding of Distractor Color Necessary for Priming Color-Singleton Search?}

It is known that precuing the target location with an onset cue reduces interference effects from distractors in the vicinity (e.g., Eriksen \& Hoffman, 1972; Paquet \& Lortie, 1990; Yantis \& Johnston, 1990). Evidence from neurophysiologicalstudies (e.g., Chelazzi, Duncan, Miller, \& Desimone, 1998; Desimone, Wessinger, Thomas, \& Schneider, 1990; Luck, Chelazzi, Hillyard, \& Desimone, 1997; Moran \& Desimone, 1985; Reynolds, Chelazzi, \& 

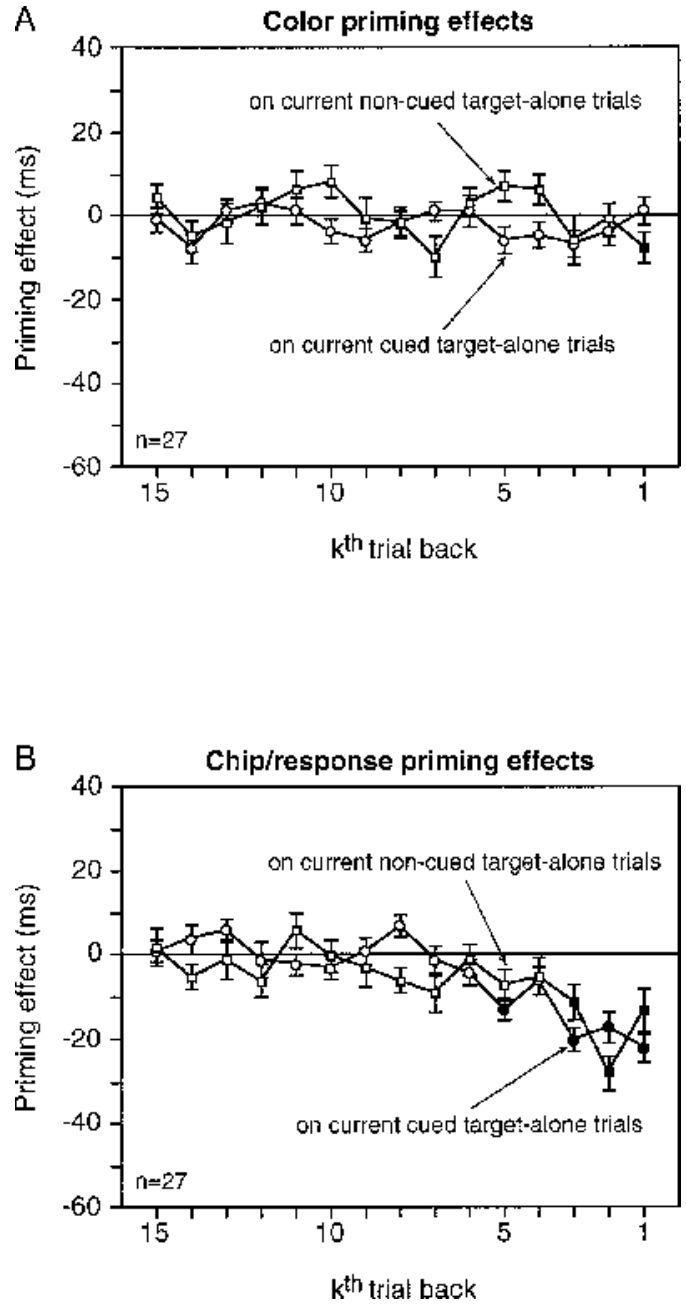

Figure 6. Color priming effects and chip/response priming effects on target-alone trials obtained in Experiment 1C. (A) Color priming effects on current noncued target-alone trials (squares) and current cued target-alone trials (circles); the priming effects have been collapsed over those produced by prior noncued trials and prior cued trials. (B) Chip/response priming effects; the format is the same as in A. Filled symbols indicate that the priming effect was significantly different from zero $(p<.05$, two-tailed). Error bars represent $\pm 1 S E$ (observers as the random effect).

Desimone, 1999) also indicates that cells in areas V2, V4, and IT (thought to be involved in visual form processing; e.g., Mishkin, Ungerleider, \& Macko, 1983; Tanaka, 1996) respond on the basis of the attended stimulus, while largely failing to respond to an unattended stimulus also present in the cell's receptive field. It is thus possible that the precuing used in Experiment $1 \mathrm{~A}$ enhanced encoding of the target color, while it reduced encoding of the distractor color. If encoding of the target color primarily produced the priming effect, this would explain why precuing the target location little affected the priming effect on a subsequent color-singleton search.

We examined this possibility by replacing the precued trials with target-alone trials (no distractors as in Exper- iment 1C). If encoding of the target color primarily drove the priming effect, the prior target-alone trials should produce strong priming effects on subsequent colorsingleton search trials. For example, a response to a red target presented among green distractors should be faster when preceded by a trial in which a single red target was presented than when preceded by a trial in which a single green target was presented. Alternatively, it is possible that encoding of distractor colors was critical, but distractor colors were encoded automatically even when the target location was precued in Experiment 1A. If this were the case, the prior target-alone trials in Experiment 2 should produce little priming on subsequent color-singleton search trials.

\section{Method}

Observers. Four observers (the 2 authors and 2 naive observers) participated.

Stimuli and Procedure. The procedure of this experiment was identical to that of Experiment 1A, except that target-alone trials, rather than cued trials, were intermixed with search trials. Observers completed 2,000-4,800 trials.

\section{Results and Discussion}

RTs were significantly faster on the target-alone trials ( $M=458 \mathrm{msec}, S E=3 \mathrm{msec})$ than on the search trails $(M=548 \mathrm{msec}, S E=2 \mathrm{msec})$ [ $t(3)=11.51, p<.005]$. This result verified that eliminating distractors substantially speeded performance.

Color priming from prior search trials and prior target-alone trials on subsequent search trials. As is shown in Figure 7A, prior search trials produced reliable priming effects on subsequent search trials. The priming effect lasted five trials (filled squares in Figure 7A) [1 st trial back, $t(3)=6.201, p<.01 ; 2$ nd trial back, $t(3)=$ $4.480, p<.05 ; 3$ rd trial back, $t(3)=4.987, p<.05 ; 4$ th trial back, $t(3)=4.182, p<.05$; and 5 th trial back, $t(3)=4.821, p<.05]$. In contrast, the prior target-alone trials produced no reliable color priming effects on subsequent search trials [for all trial lags, $t \mathrm{~s}(3)<1.913$, n.s.]. This pattern of results was obtained for all 4 observers. ${ }^{11}$ We thus infer that the validly cued trials in Experiment $1 \mathrm{~A}$ produced persistent priming, not because encoding of the target color mostly drove the priming effect, but because the distractor color was encoded regardless of whether or not observers' attention was prefocused at the target location.

Color priming from prior search trials and prior target-alone trials on subsequent target-alone trials. Little priming was obtained on a current target-alone trial whether it was preceded by a search trial or by a targetalone trial (Figure 7B). Only when immediately preceded (1 st trial back) by a target-alone trial (circles in Figure 7B) was there a small $(3 \mathrm{msec})$ yet statistically significant priming effect $[t(3)=4.503, p<.05]$. The fact that little priming was obtained for current target-alone trials replicated Experiment $1 \mathrm{C}$ and was consistent with the idea that priming facilitated the process of directing attention to the color-singleton target among distractors. As dis- 
cussed above, on a target-alone trial, the target would strongly capture attention by virtue of being the only onset item in the display.

Is color-singleton priming due to a benefit of color repetition or a cost of color switching? The properties of the target-alone trials provided a means of teasing apart the two components of the priming effect-RT benefit and RT cost. Because the prior target-alone trials did not produce color priming on subsequent search trials (with observers performing the same shape discrimination as in a search trial), prior target-alone trials provided a measure of baseline priming, which was solely due to observers' performing a particular shape discrimination task in a preceding trial. This baseline priming
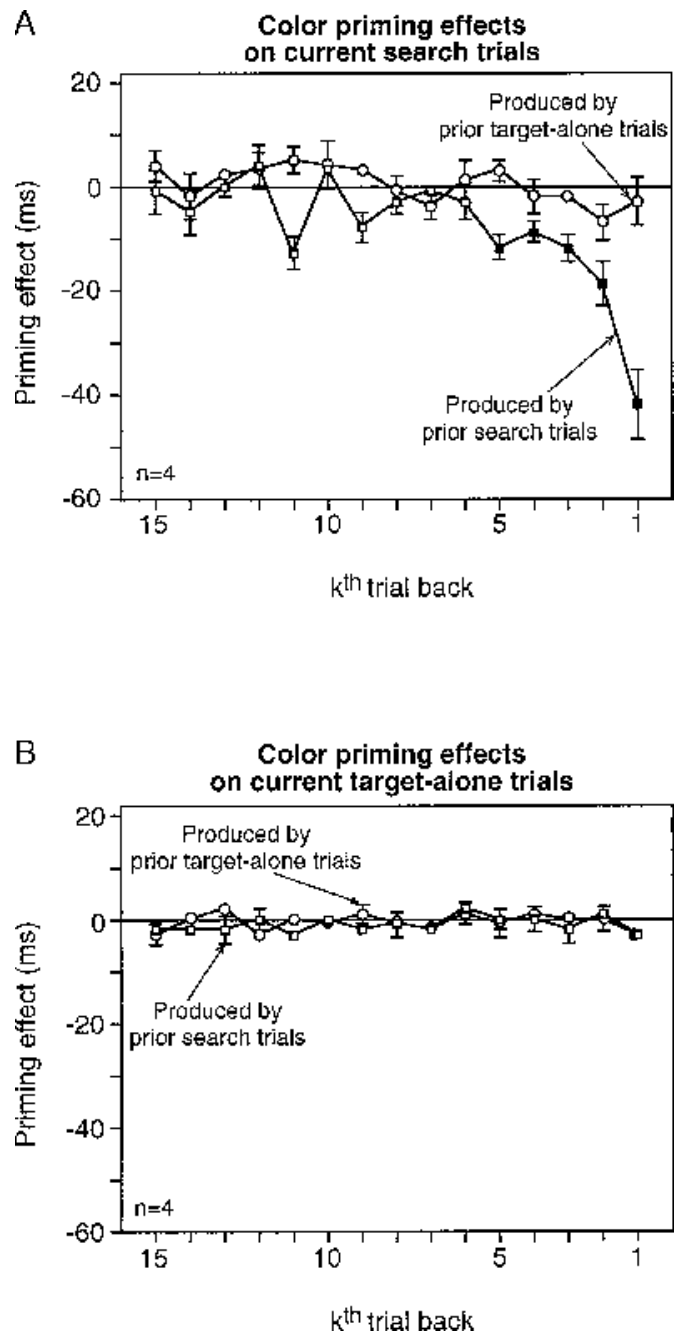

Figure 7. Color priming effects obtained in Experiment 2. (A) Priming effects on current search trials produced by prior search trials (squares) and prior target-alone trials (circles). (B) Priming effects on current target-alone trials produced by prior search trials (squares) and prior target-alone trials (circles). Filled symbols indicate that the priming effect was significantly different from zero $(p<.05$, two-tailed). Error bars represent $\pm 1 S E$ (observers as the random effect). could be used to evaluate the benefit of color repetition and the cost of color switching in the color priming effect. To this end, we compared the mean RTs on the current search trials under the following three conditions: (1) immediately preceded by a color-different search trial, (2) immediately preceded by a target-alone trial, and (3) immediately preceded by a color-same search trial. The effects of immediately preceding trials were examined because the color priming, when present, was consistently strongest at the 1st trial back. As is shown in Figure 8, the mean RT on search trials was significantly faster when preceded by a color-same search trial than when preceded by a target-alone trial $[524 \mathrm{msec}$ vs. $551 \mathrm{msec}, t(3)=12.216, p<.005]$, but significantly slower when preceded by a color-different search trial than when preceded by a target-alone trial [566-msec vs. $551 \mathrm{msec} ; t(3)=3.409, p<.05]$. All 4 observers showed this pattern of results. Thus, the priming of color-singleton search consisted of comparable contributions from both an RT benefit of color repetition and an RT cost of color switching.

In Experiment 3, we investigated whether the priming of color-singleton search was a consequence of a passive repetition priming of color-singleton perception, or required that observers attend to the color singleton at encoding.

\section{EXPERIMENT 3 \\ Is Attending to the Color-Singleton Item Necessary at Encoding?}

The results so far suggest that encoding of colors underlying the color-singleton priming effect was automatic. Distractor colors were encoded almost as strongly when the target location was precued as when observers used colors in finding the target. However, in all of the preceding experiments showing color-singleton priming, the observers always performed the chip-side discrimination on the color-singleton item. In other words, the color-singleton item was always the target item to be attended. Thus, it is not clear whether the color configuration of a color singleton display was encoded automatically or observers needed to attend to the color-singleton item. For example, an observer's having seen a red singleton among green distractors might facilitate detection of a red singleton among green distractors in a subsequent trial regardless of whether the red singleton or one of the green items was the target to be attended in the preceding trial. Alternatively, the priming of color-singleton search might require that the color singleton be the target to be attended in the preceding trial.

We tested these alternative hypotheses by precuing a nonsingleton item in half of the trials. Thus, whenever a color-singleton display was preceded by a precue, observers performed the chip-side discrimination on one of the nonsingleton items-nonsingleton-targettrials. When there was no precue, observers performed the chip-side discrimination on the color-singleton item; these trials 


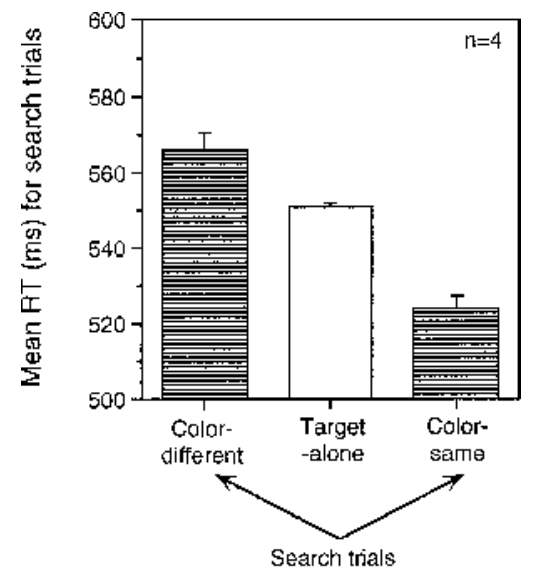

Immediately preceding trial

Figure 8. An analysis of mean RTs for Experiment 2. Mean RTs for search trials are plotted for three conditions: (1) immediately preceded by a color-different search trial, (2) immediately preceded by a target-alone trial, and (3) immediately preceded by a color-same search trial (from left to right). Error bars represent $1 S E$. (The variance due to differences in baseline RT among the observers has been removed.)

have been referred to as search trials, but here we refer to them as singleton-search trials to distinguish them from the nonsingleton-target trials.

If the color-singleton priming effect was a passive repetition priming effect for seeing a color singleton display, the prior nonsingleton-target trials and singletonsearch trials would produce equivalent priming effects on subsequent singleton-search trials; note that precuing per se does not substantially interfere with the encoding process (Experiment 1A). Alternatively, if attending to the color-singleton item at encoding was critical, the prior nonsingleton-target trials should produce little color priming on subsequent singleton-search trials (i.e., colorsame and color-different cases should make no difference).

\section{Method}

Observers. Six observers (the 2 authors and 4 naive observers) participated.

Stimuli and Procedure. The design of this experiment was identical to that of Experiment 1A except that the precue was always presented at the location of a nonsingleton item; thus, on precued trials, the observers always performed the chip-side discrimination on a nonsingleton item. When no precue was presented, the observers were instructed to perform the chip-side discrimination on the color-singleton item. As in Experiment 1A, the cued trials and search trials were randomly intermixed. All observers completed 4,800 trials.

\section{Results and Discussion}

It might seem that this task would be particularly difficult because observers had to perform the chip-side discrimination on a nonsingleton item (while ignoring the salient color-singleton item) when the trial was precued but on the color-singleton item when the trial was not precued. However, we found no evidence that suggested that this experiment was any more difficult than Experiment 1A, in which the observers always performed the chip discrimination on the color-singleton item. None of the observers (including the authors) found it particularly difficult, and the error rates were low $(<3 \%$ on average). Average RTs on the (cued) nonsingleton-target trials and the singleton-search trials in this experiment (431 $\mathrm{msec}$ and $604 \mathrm{msec}$, respectively) were also comparable to the cued and search trials in Experiment $1 \mathrm{~A}$ (433 msec and $601 \mathrm{msec}$, respectively).

The effect of position-cuing on RTs. As in the preceding experiments, there was a large cuing effect. The responses on the (cued) nonsingleton-target trials $(M=$ $431 \mathrm{msec}, S E=15 \mathrm{msec}$ ) were substantially faster than those on the singleton-search trials $(M=604 \mathrm{msec}, S E=$ $31 \mathrm{msec}$ ), yielding a large cuing effect of $173 \mathrm{msec}[t(5)=$ $9.340, p<.001]$. We note that the cuing effect in this experiment (where a nonsingleton item was precued; $173 \mathrm{msec}$ ) was comparable to the cuing effect in Experiment 1A (where the color-singleton item was precued; $168 \mathrm{msec}$ ), and the baseline response times were also comparable in the two experiments. Thus, position cuing in this experiment appears to have overridden any attention-drawing effects of the irrelevant color-singleton item present at a noncued location.

Color priming from prior nonsingleton-target trials and prior singleton-search trials on subsequent singleton-search trials. As is shown in Figure 9A, the prior singleton-search trials produced persistent priming on subsequent singleton-search trials. The priming effect lasted six trials (filled squares in Figure 9A) [1st trial back, $t(5)=5.779, p<.01 ; 2$ nd trial back, $t(5)=4.026$, $p<.05$; 3rd trial back, $t(5)=5.396, p<.005$; 4th trial back, $t(5)=5.033, p<.005 ; 5$ th trial back, $t(5)=3.661$, $p<.05$; and 6th trial back, $t(5)=13.790, p<.0001]$. In contrast, the prior nonsingleton-target trials (in which a nonsingleton item was attended) produced no reliable color priming effects on subsequent singleton-search trials. The priming effect did not reach statistical significance at the 1 st trial back $[t(5)=1.921$, n.s.]. All 6 observers showed this pattern of results. ${ }^{12}$ A repetition of a color-singleton display thus did not facilitate detection of a color-singleton target when the singleton item was not the target item in the previous trial.

It is interesting to note that performing a color-singleton search in this experiment produced persistent priming effects on a subsequent color-singleton search trial despite the fact that observers had to ignore the colorsingleton item in half of the randomly intermixed trials. It is as if performing a color-singleton search laid a memory trace that persisted through the intervening trials in which the color singleton was ignored and exerted its influence when the task was again a color-singleton search.

Color priming effects from prior nonsingletontarget trials and prior singleton-search trials on subsequent nonsingleton-target trials. As is shown in Figure $9 \mathrm{~B}$, little priming effect was obtained on a (cued) 
nonsingleton-target trial whether it was preceded by a singleton-search trial [1 st trial back, $t(5)=0.389$, n.s.] or a nonsingleton-target trial [1st trial back, $t(5)=0.241$, n.s.]. This is consistent with the results of our preceding experiments demonstrating that color-singleton priming is absent on current position-cued trials.

\section{GENERAL DISCUSSION}

The goal of this study was to understand the encoding requirements and the mechanism of the priming of colorsingleton search. In a standard task, observers detected the color-singleton item and performed a shape discrim-
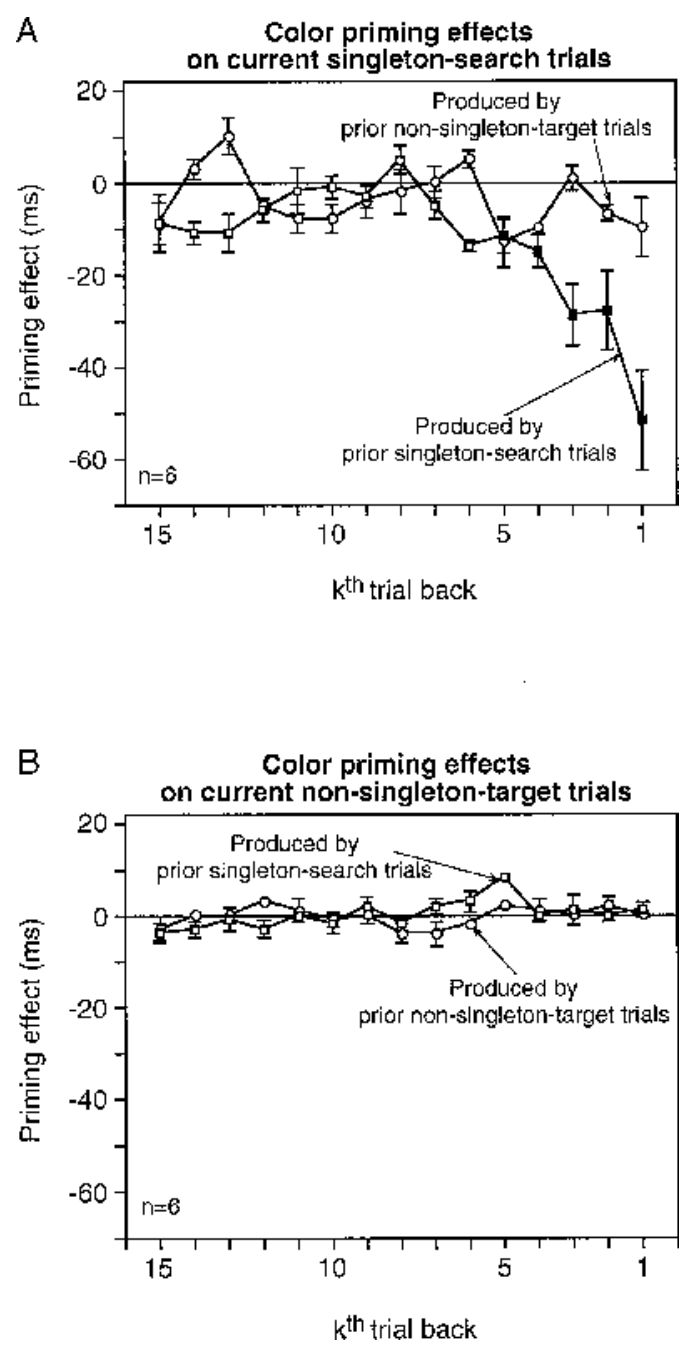

Figure 9. Color priming effects obtained in Experiment 3. (A) Priming effects on current singleton-search trials produced by prior singleton-search trials (squares) and prior nonsingletontarget trials (circles). (B) Priming effects on current nonsingletontarget trials produced by prior singleton-search trials (squares) and prior nonsingleton-target trials (circles). In nonsingletontarget trials, the target location was precued (see text for details). Filled symbols indicate that the priming effect was significantly different from zero $(p<.05$, two-tailed). Error bars represent $\pm 1 S E$ (observers as the random effect). ination on it. In order to understand which perceptual/ cognitive process(es) might be involved in this priming effect, we speculated that performing the task required the following processes: (1) detecting colored items, (2) using color to locate and direct attention to the singleton item, (3) focusing attention on the color-singleton item, (4) identifying the shape of the color-singletonitem, and (5) responding by pressing the appropriate computer key. Using this framework, we aimed to understand, first, which of these processes might be critical for encoding colors underlying the priming effect and, second, which of these processes might be facilitated by the priming effect.

Our results indicated that precuing the target location bypassed the second process, using color to locate and direct attention to the singleton item, without causing a general RT floor effect (see Experiments 1A, 1B, and 1C). Thus, if this process was required for appropriately encoding the relevant colors, prior precued trials should not produce priming effects on subsequent search trials. Similarly, if the priming effect facilitated this process, current precued trials should not be affected by the priming effect. The pattern of results we obtained was that precued trials produced reliable (though slightly reduced in magnitude) priming on subsequent search trials, but were themselves little affected by priming. We thus reasoned that the process of using color to locate and direct attention to the singleton item was not required (though it was beneficial) at encoding, but it was the process that was facilitated by priming.

The encoding processes were further examined in Experiments 2 and 3 . The results of Experiment 2 suggested that the reason precuing at encoding did not much affect priming (in Experiment 1A) was not that the repetition of the target color primarily carried the priming effect, but that the distractor color was encoded even when the target location was precued. Experiment 3 then showed that the third process, focusing attention on the colorsingleton item, was required at encoding in order to facilitate color-singleton search on subsequent trials. Note that this encoding requirement imparts a potentially ecologically beneficial feature to the priming effect; priming would facilitate the process of directing attention to a color-singleton item only if the color singleton was an important item (to be attended) in the preceding trial. In short, our results suggest that when a color-singleton item happens to be the focus of attention in one situation, a color singleton defined by the same color contrast is detected faster in subsequent encounters (lasting up to six trials or $\sim 17 \mathrm{sec}$ in this study).

An important follow-up question is whether the results we obtained for the priming of color-singleton search generalize to the priming of other types of singleton search, such as the priming of orientation-singletonsearch (Hillstrom, 2000) and the priming of spatial-frequencysingleton search (Maljkovic \& Nakayama, 1994). On the one hand, it is possible that the visual system automatically encodes a useful singleton context whenever a contrast in a particular feature (e.g., color, luminance, orientation, size/spatial frequency) happens to distinguish the 
attended item; consequently, subsequent searches on the basis of the same feature contrast are facilitated. On the other hand, a color singleton may have a special status; the priming of singleton searches on the basis of other features might not be as automatic and may require that observers attend to the relevant features in finding the target at encoding. It is apparent from just looking out a window that differences in color (rather than differences in orientation and spatial frequency) provide prevalent cues for object identity and for determining which parts of the visual image belong to same objects.

\section{Relation to Other Studies}

The finding that encoding of the distractor color was not much affected by effectively precuing the target location is generally consistent with previous studies that have found that focusing attention on the target item(s), and away from the distractor item(s), reduced interference from the distractors but did not weaken encoding of the distractors. For example, Fox $(1994,1995)$ used a letter identification task in which observers identified (via keypress) the target letter (indicated by a bar marker next to it) while ignoring the distractor letter. When the distractor letter in the prime trial became the target letter in the following probe trial, the identification latency was slowed; this type of slowing of RT for a previously ignored (or inhibited) item is generally referred to as $n e g$ ative priming (e.g., Tipper, 1985). One of the critical manipulations in Fox's studies was that, on some of the prime trials, the target location was precued by an onset cue. Despite the fact that the precuing reduced the distractor interference for the prime trial (presumably by focusing attention at the target location), it did not reduce the amount of negative priming when the distractor item became the target in the subsequent probe trial. The result thus suggested that encoding of the distractor item (as measured by a negative priming effect) was not weakened by effectively precuing the target location (but see Paquet, 2001). A similar conclusion was reached by Driver and Tipper (1989), who reported that encoding of the distractor digits underlying their negative priming effect was comparable whether those digits were presented at attended locations or at unattended locations.

These results raise the possibility that negative priming effects on the distractors might be the underlying mechanism of the priming of color-singleton search. Because the distractors were ignored on the basis of their color while observers focused their attention on the target (in performing the chip-side discrimination), a negative priming effect might make the same-colored distractors appear less salient when they are repeated in subsequent trials. Such a color-based "inhibition" of the distractors could facilitate target detection, for example, by making the target appear more salient. There are, however, other possibilities. For example, rapid grouping of the distractors into a uniform same-colored region would facilitate the process of detecting the odd-colored target (e.g., see Duncan \& Humphreys, 1989, for a review). This grouping process might be speeded when the same-colored distractors are repeated; for example, when green distractors were grouped as distractors in one trial, the green color might get tagged as the to-be-grouped color, thereby facilitating the process of distractor grouping in a subsequent trial if the distractors were green again. These two mechanisms might both contribute. Further research is thus needed to understand the mechanism by which priming facilitates the process of directing attention to the singleton item.

To place our results in a broader context, previous research has developed models describing the two main types of sequential priming effects that influence RT, one due to repetitions of the stimulus feature to which visual discrimination is made, stimulus-repetition priming, and the other due to repetitions of the same response, responserepetition priming. These two types of priming have been empirically dissociated typically by mapping multiple stimuli to each response finger (information-reduction paradigm; see Bertelson, 1965; Rabbitt, 1968; Smith, 1968 , for early references). The stimulus-repetition priming effects (over and above the response-repetition priming effects) have been assessed by comparing RTs when the stimulus and response were both repeated with RTs when only the response was repeated. The responserepetition priming effects (over and above the stimulusrepetition priming effects) have been assessed by examining cases in which the same response was repeated (versus not repeated) while the stimulus changed.

Stimulus-repetition priming is more prevalent than response-repetition priming and is thought to occur due to the strengthening of the mapping from the specific stimulus representations to the corresponding motor responses (e.g., Pashler \& Baylis, 1991, response-selection shortcut). Response-repetition priming is thought to occur due to the strengthening of the mapping from the category representations of the to-be-identified stimuli to the corresponding motor responses. The primary evidence for this idea is that response-repetition priming (across different stimuli) only occurs when the stimuli from the same category (e.g., letters, digits, and symbols) are consistently mapped to the same response and does not occur when multiple stimulus categories are mapped to each response (e.g., Campbell \& Proctor, 1993; Pashler \& Baylis, 1991). Response-repetition priming is also known to be affected by observers' expectation (see Soetens, 1998, for a review).

Our results complement these theories that model priming effects as being mediated by modifications in the information processing pathways that connect the processes of stimulus identification, stimulus categorization, and response selection. Our present finding adds "early" priming effects that occur at the stage of stimulus selection that precedes stimulus identification. Consistent with this idea, we demonstrated that priming of stimulus selection can be functionally dissociated from priming of stimulus identity and response. The onset position cue, which preempted the target-selection process but was uninformative for the processes of identifying the chip side and selecting an appropriate response, eliminated 
the color-based priming effect on the current cued trials, but left the chip/response priming effect unaffected.

To conclude, our results suggest that salient visual contexts that distinguish an attended item (such as the target's being a color-singleton) are encoded automatically. Repetitions of the same context can then facilitate the process of directing attention to the previously attended item in subsequent trials (up to six trials or $\sim 17 \mathrm{sec}$ ).

\section{REFERENCES}

Bertelson, P. (1963). S-R relationships and reaction times to new versus repeated signals in a serial task. Journal of Experimental Psychology, 65, 478-484.

Bertelson, P. (1965). Serial choice reaction-time as a function of response versus signal-and-response repetition. Nature, 206, 217-218.

Bravo, M. J., \& NaKay ama, K. (1992). The role of attention in different visual-search tasks. Perception \& Psychophysics, 51, 465-472.

Campbell, K. C., \& Proctor, R. W. (1993). Repetition effects with categorizable stimulus and response sets. Journal of Experimental Psychology: Learning, Memory, \& Cognition, 19, 1345-1362.

Cheal, M., \& Lyon, D. (1989). Attention effects on form discrimination at different eccentricities. Quarterly Journal of Experimental Psychology, 41A, 719-746.

Chelazzi, L., Duncan, J., Miller, E. K., \& Desimone, R. (1998). Responses of neurons in inferior temporal cortex during memory-guided visual search. Journal of Neurophysiology, 80, 2918-2940.

Desimone, R., Wessinger, M., Thomas, L., \& Schneider, W. (1990). Attentional control of visual perception: Cortical and subcortical mechanisms. Cold Spring Harbor Symposia on Quantitative Biology, 55, 963-971.

Di Pace, E., Marangolo, P., \& Pizzamiglio,L. (1997). Response bias in color priming. Acta Psychologica, 95, 3-14.

Driver, J., \& TiPPER, S. P. (1989). On the nonselectivity of "selective" seeing: Contrasts between interference and priming in selective attention. Journal of Experimental Psychology: Human Perception \& Performance, 15, 304-314.

Duncan, J., \& Humphreys, G. W. (1989). Visual search and stimulus similarity. Psychological Review, 96, 433-458.

Egeth, H. E., \& YANTIS, S. (1997). Visual attention: Control, representation, and time course. Annual Review of Psychology, 48, 269-297.

Entus, A., \& Bindra, D. (1970). Common features of the "repetition" and "same-different" effects in reaction time experiments. Perception \& Psychophysics, 7, 143-148.

Eriksen, C. W., \& Collins, J. F. (1969). Temporal course of selective attention. Journal of Experimental Psychology, 80, 254-261.

ERIKsen, C. W., \& Hoffman, J. E. (1972). Temporal and spatial characteristics of selective encoding from visual displays. Perception \& Psychophysics, 12, 201-204.

ERIKSEn, C. W., \& Rohrbaugh, J. W. (1970). Some factors determining efficiency of selective attention. American Journal of Psychology, 83, 330-342.

Fox, E. (1994). Interference and negative priming from ignored distractors: The role of selection difficulty. Perception \& Psychophysics, 56, 565-574.

Fox, E. (1995). Pre-cuing target location reduces interference but not negative priming from visual distractors. Quarterly Journal of Experimental Psychology, 48A, 26-40.

Hillstrom, A. P. (2000). Repetition effects in visual search. Perception \& Psychophysics, 62, 800-817.

Kornblum, S. (1967). Choice reaction time for repetitions and nonrepetitions: A re-examination of the information hypothesis. Acta Psychologica, 27, 178-187.

Kröse, B. J. A., \& Julesz, B. (1989). The control and speed of shifts of attention. Vision Research, 29, 1607-1619.

LaBerge, D., Legrand, R, \& Hobbie, R. K. (1969). Functional identification of perceptual and response biases in choice reaction time. Journal of Experimental Psychology, 79, 295-299.

LaBerge, D., Van Gelder, P., \& Yellott, J., Jr. (1970). A cueing technique in choice reaction time. Perception \& Psychophysics, 7, 57-62.
Luck, S. J., Chelazzi, L., Hillyard, S., \& Desimone, R. (1997). Neural mechanisms of spatial selective attention in areas V1, V2, and V4 of macaque visual cortex. Journal of Neurophysiology, 77, 24-42.

Mackeben, M., \& NaKayama, K. (1993). Express attentional shifts. Vision Research, 33, 85-90.

Maljkovic, V., \& Nakay ama, K. (1994). Priming of pop-out I: A role of features. Memory \& Cognition, 22, 657-672.

Maljkovic, V., \& Nakayama, K. (2000). Priming of pop-out: III. A short-term implicit memory system beneficial for rapid target selection. Visual Cognition, 7, 571-595.

Mishkin, M., Ungerleider,L. G., \& Macko, K. A. (1983). Object vision and spatial vision: Two central pathways. Trends in Neurosciences, 6, 414-417.

Moran, J., \& Desimone, R. (1985). Selective attention gates visual processing in the extrastriate cortex. Science, 229, 782-784.

Nakayama, K., \& Mackeben, M. (1989). Sustained and transient components of focal visual attention. Vision Research, 29, 1631-1647.

PAQuet, L. (2001). Eliminating flanker effects and negative priming in the flankers task: Evidence for early selection. Psychonomic Bulletin \& Review, 8, 301-306.

Paquet, L., \& Lortie, C. (1990). Evidence for early selection: Precuing target location reduces interference from same-category distractors. Perception \& Psychophysics, 48, 382-388.

Pashler, H., \& Baylis, G. (1991). Procedural learning: 2. Intertrial repetition effects in speeded-choice tasks. Journal of Experimental Psychology: Learning, Memory, \& Cognition, 17, 33-48.

RaвbitT, P. M. A. (1968). Repetition effects and signal classification strategies in serial choice-response tasks. Quarterly Journal of Experimental Psychology, 20, 232-239.

Reynolds, J. H., Chelazzi, L., \& Desimone, R. (1999). Competitive mechanisms subserve attention in macaque areas V2 and V4. Journal of Neuroscience, 19, 1736-1753.

Rovamo, J., \& VIrSU, V. (1979). Visual resolution, contrast sensitivity, and the cortical magnification factor. Experimental Brain Research, 37, 475-494.

Shulman, G. L., Remington, R. W., \& McLean, J. P. (1979). Moving attention through physical space. Journal of Experimental Psychology: Human Perception \& Performance, 5, 522-526.

Simon, J. R. (1988). A 'priming' effect in a choice reaction time task. Acta Psychologica, 69, 45-60.

Sмiтн, M. C. (1968). Repetition effect and short-term memory. Journal of Experimental Psychology, 77, 435-439.

SoEtens, E. (1998). Localizing sequential effects in serial choice reaction time with the information reduction procedure. Journal of Experimental Psychology: Human Perception \& Performance, 24, 547 568.

Suzuki, S., \& CAVAnagh, P. (1997). Focused attention distorts visual space: An attentional repulsion effect. Journal of Experimental Psychology: Human Perception \& Performance, 23, 443-463.

TANAKA, K. (1996). Inferotemporal cortex and object vision. Annual Review of Neuroscience, 19, 109-139.

Theeuwes, J. (1991). Cross-dimensional perceptual selectivity. Perception \& Psychophysics, 50, 184-193.

Theeuwes, J. (1992). Perceptual selectivity for color and form. Perception \& Psychophysics, 51, 599-606.

TIPPER, S. P. (1985). The negative priming effect: Inhibitory effects of ignored primes. Quarterly Journal of Experimental Psychology, 37A, 571-590.

Treisman, A. M., \& Gelade, G. (1980). A feature-integration theory of attention. Cognitive Psychology, 12, 97-136.

UlRich, R, \& MATtes, S. (1996). Does immediate arousal enhance response force in simple reaction time? Quarterly Journal of Experimental Psychology, 49A, 972-990.

Yantis, S., \& Johnston, J. C. (1990). On the locus of visual selection: Evidence from focused attention tasks. Journal of Experimental Psychology: Human Perception \& Performance, 16, 135-149.

\section{NOTES}

1. Frequent repetitions of the same response (e.g., pressing the right response key with right index finger) can speed up response time for 
that response relative to a less frequent alternative response (e.g., pressing the left response key with left index finger) by biasing the observer toward making the more frequent response (e.g., LaBerge, Legrand, \& Hobbie, 1969; LaBerge, Van Gelder, \& Yellott, 1970).

2. Hillstrom (2000) used a similar paradigm and found that the color priming effect lasted only one trial (if evaluated using the sequential $t$ test method discussed below) when she tested college undergraduate students. Hillstrom thus suggested that Maljkovic and Nakayama (1994) obtained long-lasting effects because they used highly experienced observers who might have developed a specific strategy. However, when we conducted an experiment similar to Maljkovic and Nakayama's (1994) using inexperienced underg raduate students $(N=30 ; 450$ trials per observer), we obtained fairly long-lasting priming effects (lasting through four intervening trials; unpublished data). Therefore, it is likely that the short-lasting priming effects obtained by Hillstrom are specific to the stimulus parameters she used, rather than due to the fact that inexperienced observers use different strategy than experienced observers.

3 . Whenever each observer was tested extensively (all experiments except Experiment 1C), consistent patterns of results were obtained from all observers. (Large numbers of trials allowed us to see the experimental effects clearly in each observer's data.) The fact that those who participated in multiple experiments as well as those who participated in only one experiment produced the same patterns of results suggests that the results reported here are robust.

4. As Maljkovic and Nakayama (1994) points out, the shape discrimination made in this task is a relatively high-acuity judgment. Previous work has shown that acuity diminishes as a function of visual eccentricity faster along the vertical axis than along the horizontal axis (Kröse \& Julesz, 1989; Rovamo \& Virsu, 1979). We thus adopted the horizontally elongated ellipse used by Maljkovic and Nakayama (1994) to approximately equalize the acuity at all possible target positions.

5 . With a relatively small number of trials per condition per observer, we thought it appropriate to use medians to represent central tendencies for each observer in this case. However, the results are virtually unchanged even when means are used instead.

6 . We note that the magnitude of the chip/response priming effects obtained in this task tend to be small (undetected by Maljkovic \& Nakayama, 1994). A possible reason is that the S-R compatibility for the twochoice chip-side discrimination task was highly optimal and simple (right index finger in the right position being used to indicate the rightsided chip and left index finger in the left position being used to indicate the left-sided chip). It has been known that stimulus/response priming is smaller when S-R compatibility is more optimal or simpler (e.g.,
Bertelson, 1963; Entus \& Bindra, 1970), and when the response has only two choices as opposed to multiple choices (e.g., Kornblum, 1967).

7. Detailed investigation of the chip/response priming effects (e.g., the reason why they might be longer lasting for current cued trials) is outside the scope of this article.

8. As pointed out by one of the reviewers, this result does not rule out the possibility that the cue interferes with encoding of color when it is presented at the location of the target item (possibly via local masking), but not when it is presented elsewhere.

9. In this experiment, we opted to test a large number of inexperienced observers in a modest number of trials (200) rather than testing a small number of observers extensively. To determine whether or not the chip/ response priming effects diminished on the current cued trials (relative to the current noncued trials), we needed to obtain robust chip/response priming effects at least on the current noncued trials. As chip/response priming effects were undetected by Maljkovic and Nakayama (1994) using highly trained observers, we reasoned that we would increase the chip/response priming effect by testing inexperienced observers.

10. Each observer was also tested in another set of trials in which the target position was fixed during each block of trials. The data from those fixed-position trials will not be presented here because those trials produced the same pattern of results as the random-position trials did (with regard to the purpose of this experiment).

11. When a two-way repeated measures analysis of variance (ANOVA) was conducted using the preceding trial type (search trials or targetalone trials) and the $k$ th trial back (up to the 5 th trial back for which the priming effects on the search trials were significant) as the two factors, the main effect of the preceding trial type $[F(1,3)=61.807, p<.005]$ and the interaction between the preceding trial type and the $k$ th trial back $[F(4,12)=3.786, p<.05]$ were both significant. This confirms the trends seen in Figure 7A (the priming function produced by the search trials being of greater magnitude and steeper overall than that produced by the target-alone trials).

12. When a two-way repeated measures ANOVA was conducted with the preceding trial type (singleton-search trials or nonsingleton-target trials) and the $k$ th trial back (up to the 6 th trials back for which the priming effects on the search trials were significant) as the two factors, the main effect of the preceding trial type $[F(1,5)=23.437, p<.005]$ and the interaction between the preceding trial type and the $k$ th trial back $[F(5,25)=14.943, p<.0001]$ were both significant. This confirms the trends seen in Figure 9A (the priming function produced by the singletonsearch trials being of greater magnitude and steeper overall than that produced by the nonsingleton-target trials).

(Manuscript received December 31, 1999; revision accepted for publication December 4, 2000.) 